\title{
Design and Implementation of an Autonomous Waste Sorting Machine Using Machine Learning Technique
}

\author{
Shirazush Salekin Chowdhury, Nahian Binte Hossain, Tamal Saha, Jannatul Ferdous, Md. Saniat Rahman Zishan
}

\begin{abstract}
In a world where the population is inevitably increasing, waste produced is progressively increasing as well. In this project, an autonomous waste sorting machine was made which could detect multiple classes of waste materials, and then separate them accordingly. The waste products were taken as input in a funnel-shaped structure and dropped one by one to a conveyor belt where they would be detected by machine learning technique using Faster-RCNN, and then a servo motor would separate them according to the detection result. In rare cases, there are some misdetections of the waste materials, but the reliability of the detection was very high. Our work can facilitate human efforts to separate waste products and can make the waste sorting system completely automatic.
\end{abstract}

Index Terms-Machine Learning, Object detection, Separation of objects, TensorFlow, Convolutional Neural Networks, Inductive Proximity Sensor, Waste Sorting, Computer Vision.

\section{INTRODUCTION}

$\mathrm{W}$ aste grows with the growth of the economy and human life. Over time as it starts building up, it is crucial to remove them properly in order to maintain a hygienic society and a healthy Earth as a whole. Despite proper disposal of trash having a key role in the healthy survival of all sorts of living organisms, it has yet been neglected to a great extent.

Shirazush Salekin Chowdhury

Post-Graduate Student, Dept. of EEE

American International university-Bangladesh (AIUB)

Dhaka, Bangladesh

Email: salekinchowdhury@gmail.com

Nahian Binte Hossain

Graduate, Dept. of EEE

American International university-Bangladesh (AIUB)

Dhaka, Bangladesh

Email: nahian.b.h@gmail.com

Tamal Saha

Graduate, Dept. of EEE

American International university-Bangladesh (AIUB)

Dhaka, Bangladesh

Email: sahatamal1996@gmail.com

\section{Jannatul Ferdous}

Graduate, Dept. of EEE

American International university-Bangladesh (AIUB)

Dhaka, Bangladesh

Email: jjannatjui@gmail.com

Md. Saniat Rahman Zishan

Associate Professor, Dept. of EEE and $\mathrm{CoE}$

Head, Department of CoE

American International university-Bangladesh (AIUB)

Dhaka, Bangladesh

Email: saniat@aiub.edu
In Bangladesh, waste generation is gradually increasing. But a significant number of people are not getting the service of proper waste disposal. A substantial portion of wastes in Bangladesh is not appropriately collected. Ultimate disposal of these wastes is not done properly due to the shortage of awareness, motivation, expertise, and money [1]. Choosing an appropriate disposal spot is a significant task for the useful clearance of municipal garbage [2]. Due to the inappropriate dumping of waste materials, the most common problems are an aesthetic nuisance, disease transmission, air and water pollution, odor nuisance, and economic losses [3]. In developing countries, controlling municipal solid waste is an unavoidable challenge as in Dhaka city [4]. Incineration and landfills have been the most conventional methods for waste removal. These disposal methods have been continually polluting the environment, the air that we breathe in, adding to greenhouse gases, and above all adding to the already increasing global warming. The latter is filling up land sites, polluting the river, and the surrounding neighborhood [5]. These are all the primary reasons for which an automated waste sorting machine is designed. It is essential to sort important materials from the waste so that they can be recycled later. Metal, plastic, glass, paper, cloth, biodegradable materials, and many other waste materials can be sorted and recycled. This will lead us one step closer to a healthy, sustainable life and environment. The main objective of this work is to facilitate human efforts to separate waste products and to make the waste sorting system completely automatic. In this paper, Faster-RCNN with InceptionV2 architecture was chosen for transfer learning as the prediction time taken per image is the lowest compared to other CNN models.

\section{RELATED WORK}

Many researchers worked on the detection and separation of waste materials and found several possible solutions. Rafeeq et al [6] designed a system where the infra-red sensor is used to detect waste and start the conveyor belt. Metal is detected by the inductive proximity sensor and plastic and glass are detected by the capacitive sensor. Elfasakhany et al [7] designed a system to differentiate between glass and plastic. An air fan was used to blow away plastic materials depending on the density dissimilarities of glass and plastic. Samreen et al [8] also designed a prototype to sort metal, plastic, and glass by using the inductive proximity sensor for metal and capacitive proximity sensor for differentiating between plastic and glass. A. Madankar et al [9] designed a system where an IR sensor was used to detect the presence of waste materials 
and turning on the conveyor belt. To segregate metallic waste electromagnet was used. A blower was used so that the dry wastes could be blown off to a container specified for the dry materials. The rest of the materials are of wet wastes and they were eventually fallen into another container. Andres et al [10] used a machine learning method where an RGB image is converted to a grayscale image and then into a binary image for image processing. After that, characteristic extraction is done followed by machine learning to train data. Sharanya et al [11] developed a system where a moisture sensor is used for wet waste, the inductive proximity sensor is used to detect metal, a combination of laser and LDR is used to differentiate between plastic and paper. Plastic will permit the laser light, unlike paper which will not let the laser pass through. Sudha et al [12] developed a system where deep learning is used. R. Laszlo et al [13] developed a sorting system where a contour vision sensor was for acquiring, processing, and recognizing the captured images. M. S. Haque Sunny et al [14] developed Region-based Convolutional Neural Networks (R-CNN) based image classifier for detecting and recognizing objects by analyzing training features. Szegedy, C. et al proposed FasterRCNN with InceptionV2 architecture for transfer learning as prediction time taken per image is the lowest compared to other CNN models [15] [16].

\section{ARCHITECTURE AND DESIGN OF THE SYSTEM}

The autonomous waste sorting machine is designed and implemented using both sensors and machine learning methods. A funnel-shaped mechanism is used to collect waste materials in bulk and deploy on the conveyor belt one by one. A conveyor is used to carry waste materials from one end to another end. To use the power efficiently conveyor will only run whenever it detects an object by using an IR sensor. The metallic object was identified by employing an inductive proximity sensor. For the rest of the waste products, i.e. plastic bottles, glasses, paper, etc. machine learning is used. For machine learning, TensorFlow is used which is an end-to-end open-source library for numerical computation. A Faster RCNN object detection algorithm is used to detect objects. A computer is used as a processing unit to process the real-time videos taken by the camera mounted in the conveyor. After detecting the objects boxes, and point at the middle of the detected object will appear. Serial communication is established between an Arduino and computer to pass the detected output to the Arduino from a computer. When an object is detected, a signal will come from a computer to Arduino via serial communication and that controls the servo motors. Step by step block diagram of the autonomous waste sorting machine is shown in fig. 1.

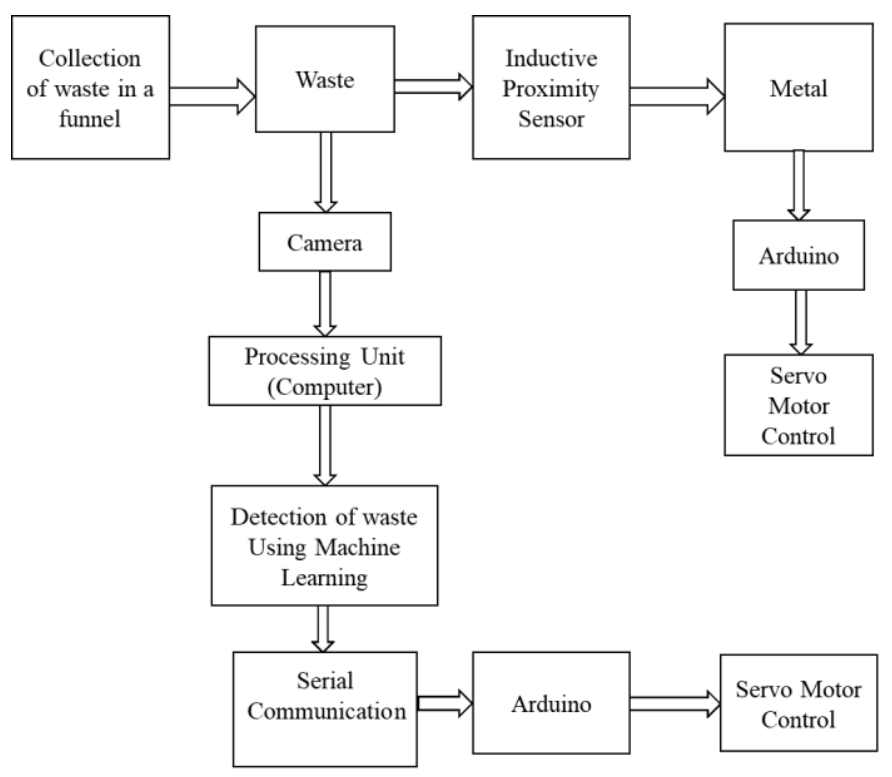

Fig. 1. Step by step block diagram of the autonomous waste sorting machine

\section{A. Transfer Learning with TensorFlow}

Transfer learning is a very important technique where a model that is already trained is utilized for fine-tuning and extraction of feature maps to train new data of specific tasks [17]. The primary advantage of using transfer learning is that the computational time needed for training the network is lesser compared to training it from scratch as the starting point of the training is a pre-trained model [18]. Therefore, for transfer learning, there are only two straightforward processes [17]:

i) The initialization of the training model with weights from a pre-trained model

ii) Training of the model using the new custom data.

\section{B. Faster-RCNN Algorithm}

For object detection, Faster-RCNN uses a single, unified network [19]. An image is taken as input that is given to a convolution layer that returns feature maps for the image. These feature maps will then be applied to the Region Proposal Network (RPN). RPN predicts a large number of possible regions where it is most likely to find the object of interest. These regions of proposals are then fed into what is essentially a Fast R-CNN. To bring down all the predicted region of proposals to the identical dimension, Region of Interest (RoI) pooling layer is used. To categorize the anticipated bounding-boxes, a Fully-Connected (FC) layer is employed where these proposals are given. In addition to the FC layer, the classification layer and bounding box regressor layers are used. In this sense, it can be said that the Faster RCNN has consisted of RPN and Fast R-CNN [20]. Fig. 2 shows the steps of Faster-RCN. 


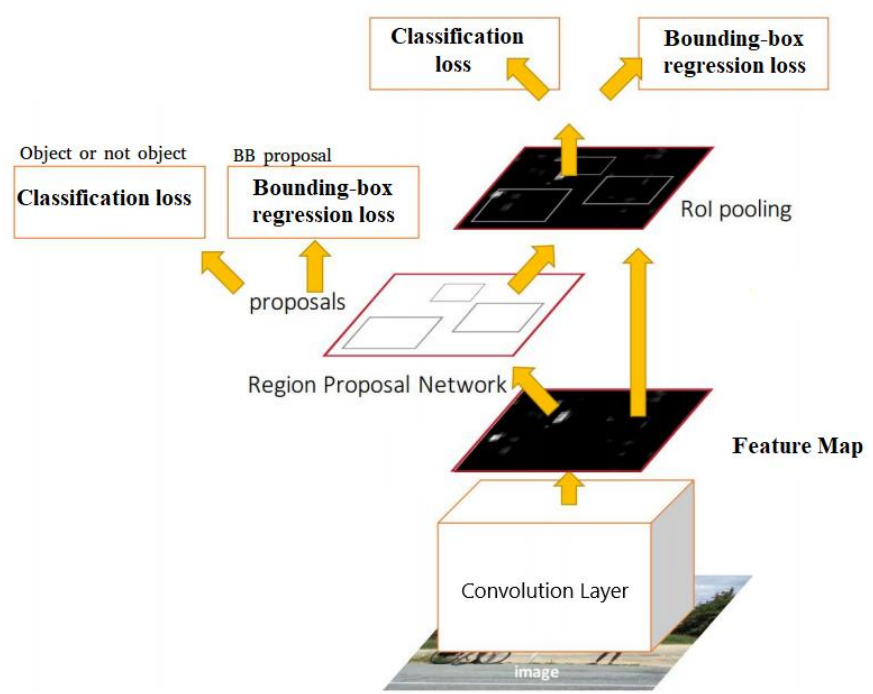

Fig. 2. Faster-RCNN object detection steps [19]

\section{EXPERIMENTAL SETUP FOR OBJECT DETECTION}

TensorFlow object detection API (Application Programming Interface) was used to create and train a custom model to detect different types of waste materials by making use of transfer learning. It is an open-source framework that makes it straightforward to build, train as well as setup object detection models. This is tremendously advantageous since creating an object detection model from scratch can be challenging and can take massive computing power [21]. For the detection of the waste materials, Faster-RCNN based InceptionV2 COCO model was used. This model has a speed of 58 milliseconds and COCO mAP (mean Average Precision) of 28 [22] [24]. COCO mAP measures the correctness of the object detector model [23]-[24]. To decrease the complexity of $\mathrm{CNN}$, the InceptionV2 module was used. CNN computational time also decreased by using this module [24]. The steps which are followed to fine-tune and transfer learning the custom object detection model for different types of waste materials with respect to the pre-trained Faster-RCNN with InceptionV2 architecture are given below:

\section{A. Preparation of Training Dataset}

Images for four different types of waste materials, namely, paper, plastic, cloth, and glass were used to prepare the training dataset. In total 300 pictures were captured in various lighting conditions and orientations. For the training dataset, eighty percent of the pictures were taken. For the testing dataset, the rest of the twenty percent photos were kept. It is important to split the total image dataset into two sub datasettrain and test datasets in order to eliminate overfitting or under-fitting of the custom model. The training dataset was used to build the custom object detection model. The test dataset was then used to verify the detection precision of the custom model [25]. The total quantity of pictures employed for training and testing purposes is shown in table I.
TABLE I. TOTAL QUANTITY OF PICTURES PER CATEGORY

\begin{tabular}{|c|c|c|c|}
\hline \multirow{2}{*}{ Categories } & \multicolumn{3}{|c|}{ The total quantity of pictures } \\
\cline { 2 - 4 } & Training & Test & Total \\
\hline Paper & 48 & 12 & 60 \\
\hline Plastic & 48 & 12 & 60 \\
\hline Cloth & 48 & 12 & 60 \\
\hline Paper & 48 & 12 & 60 \\
\hline Altogether & 48 & 12 & 60 \\
\hline Total & 240 & 60 & 300 \\
\hline
\end{tabular}

\section{B. Labeling Data}

Images of various classes of waste materials were taken. It is important to label the images and that was done by using a graphical image annotation tool called 'labelImg' [26] [32]. It is used to generate "XML" files in "PASCAL VOC" format [27]. XML files contained the coordinates of the rectangular boxes, height, depth, width, and the class name of the labeled images of different waste materials [24]. Fig. 3 shows the sample training image of waste materials using LabelImg software including manually drawn bounding boxes.

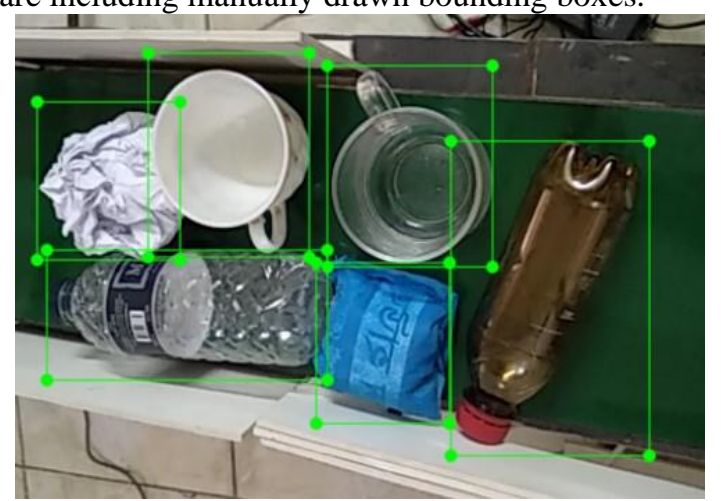

Fig. 3. Sample labeled image using labelImg software

\section{Generation of TFRecords for Training}

Generation of TFRecords was one of the vital stages for the training of the object detector as it is used as input data [28]. Information about the annotated rectangular coordinates, width, height, and the labeled names of the waste materials were kept in the TFRecod files. For generating TFRecords following steps were followed:

i) Converting XML files of training and test folders to two comma-separated values (CSV) files using a python script.

ii) Using these two CSV files of training and test, another python script was run to generate two TFRecords.

\section{Setting up the Configure File and Label Map for Training}

The label map file was used to map a given ID to a Name which would be displayed as output when the object would be detected.

The configure file contained the information about the required parameters and network architecture during training. Faster R-CNN InceptionV2 COCO config file was chosen for training. The number of steps, label map path, learning rate, fine-tune checkpoint path, the total number of classes to be detected, train and test TFRecord paths, the total number of examples are some of the significant parameters found in the config file. 


\section{E. Training the Custom Model}

After setting up the configure file correctly, a python script was run to start the training process of the custom object detection model. At the beginning of the training process, the losses per step were very high. But as the training went on, the losses were kept dropping. After the completion of training the custom model, the final step was to generate the frozen inference graph which was in Protocol Buffers/Protobuf (pb) format. This single Protobuf file contained the graph definition and variables of the checkpoint. But these hyper-parameters were saved within the graph structure as constant [29].

\section{F. Loss Graphs}

During the training, the graphs of different losses were found using TensorBoard which is a visualization toolkit of TensorFlow [30]. It was recommended to train the custom model the total loss consistently dropped below 0.05 [24] [31]. From fig. 4(a) to 4(e), there are different types of losses found from TensorBoard. A loss function is used to find out how far the anticipated values deviate from the actual values in the training data. Localization loss indicates bounding-box regressor loss. Objectness loss indicates whether the desired object is classified in the bounding-boxes or not. The loss for anticipating an output class from a set of trained classes is regarded as classification loss, for example, plastic, paper, glass, cloth, etc [33]. The total loss graph showed that around 570 steps were required for the overall loss to drop consistently below 0.05 which was shown in fig. 4(a)

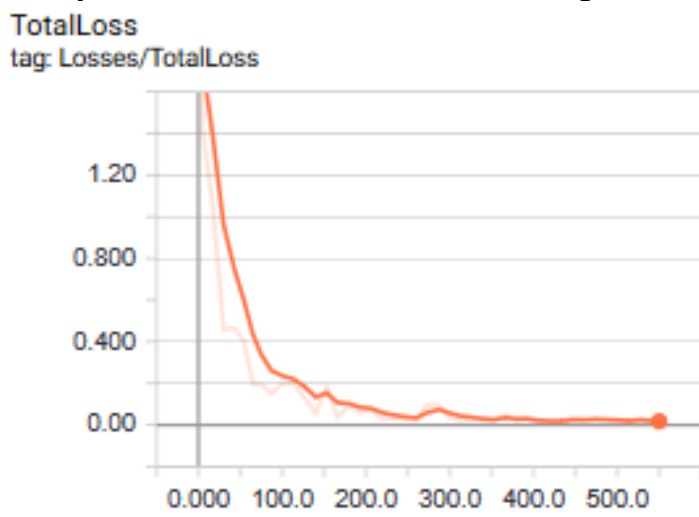

Fig. 4. (a) Total loss versus the number of steps

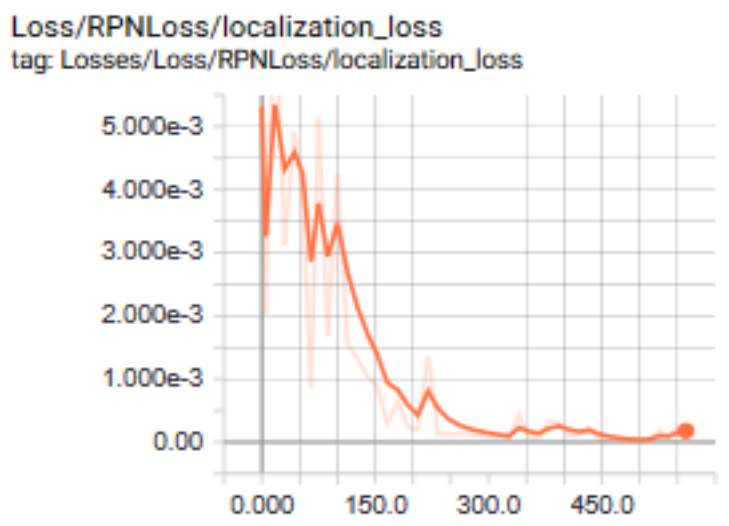

Fig. 4(b) RPN localization loss versus the number of steps
Loss/RPNLoss/objectness_loss

tag. Losses/Loss/RPNLoss/objectness_loss

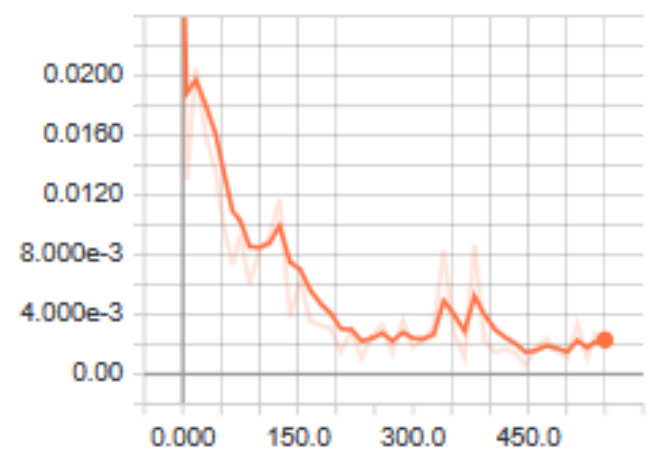

Fig. 4(c) RPN objectness loss versus the number of steps

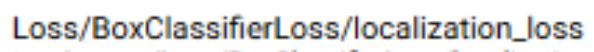

tag: Losses/Loss/BoxClassifierLoss/localization_loss

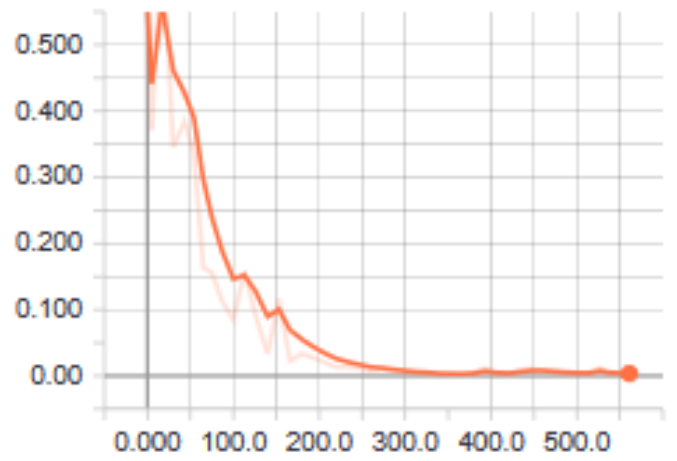

Fig. 4(d) Box Classifier localization loss versus the number of steps

Loss/BoxClassifierLoss/classification_loss tag Losses/Loss/BoxClassifierLoss/classification_loss

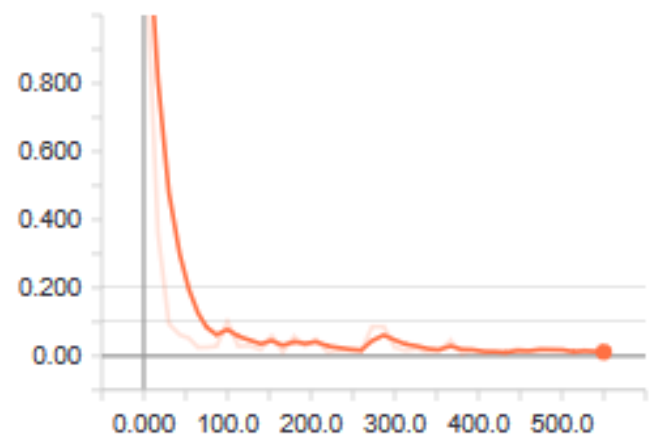

Fig. 4(e) Box Classifier classification loss versus the number of steps

\section{G. Arduino}

Arduino takes the output from both the computer and the inductive proximity sensor as its input to control the servo motors. Whenever waste material is detected by an infrared sensor, the output of the infrared sensor will be fed to an input pin of an Arduino. A motor driver will control the starting and closing of the wiper motor used to drive the conveyor belt. Whenever the Arduino gets a signal for detection of waste, it will control the corresponding servo motor to rotate its flap and block the passage of the conveyor that in turn sort out the objects. 


\section{HARDWARE DESIGN AND IMPLEMENTATION}

Every physical design needs to be drafted before implemented practically. So, CAD design is done before the implementation of the real design. The total length of the conveyor is taken as 6 feet 5 inches to accommodate all the sensors, servo motors, and a camera. After the first 12 inches, a 'square' shaped bridge is made which has a height and width of approximately 3 inches. In this square bridge, inductive proximity sensors are used to detect metallic objects. After 12.5 inches an ' $L$ ' shaped bridge is made having the same width with adjustable height. This is used for mounting the camera. CAD design of the conveyor is shown in fig. 5.

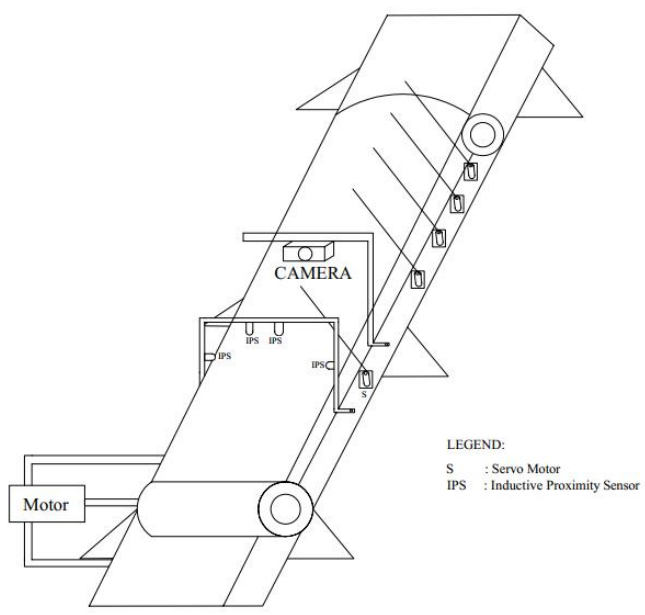

Fig. 5. CAD design of the system

A wiper motor is used to drive the conveyor belt. For the servo motors, 8 inches flap is used to work as a blockage in the passage of the conveyor after detecting the object. Each servo motor has a width of 2.1 inches. Hence, almost 10.1 inches are used for each servo motor and flaps. 5 servo motors are used. So, the total length will be 50.5 inches.

In a hardware implementation, the funnel-shaped structure is made to take multiple wastes as its input and let them fall on the conveyor belt one by one for the detection and separation of the objects. Fig. 6 shows the complete setup of the project.

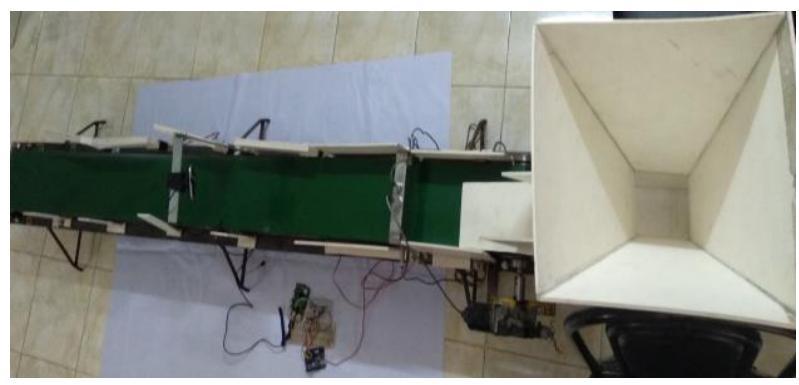

Fig. 6. The complete system to separate different waste

Fig. 7 shows the construction of a servo motor flap using PVC board which was mounted on the system.

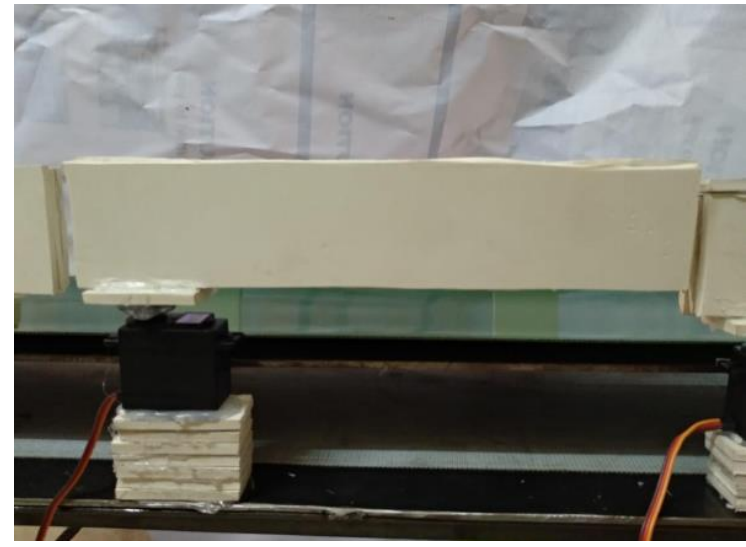

Fig. 7. Construction of servo motor flap using PVC board

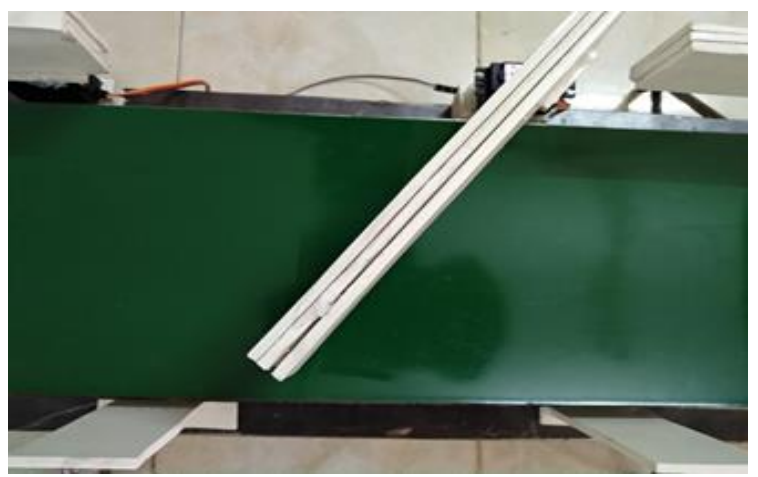

Fig. 8. The flap and the wall of the conveyor

Fig. 8 shows the closing of the gate using a servo motor after the detection of an object. A wiper motor was to drive the conveyor belt. This wiper motor could take a maximum of 5 KG load on the conveyor belt. Fig. 9 shows the implementation of the wiper motor on the system that was used to drive the conveyor belt.

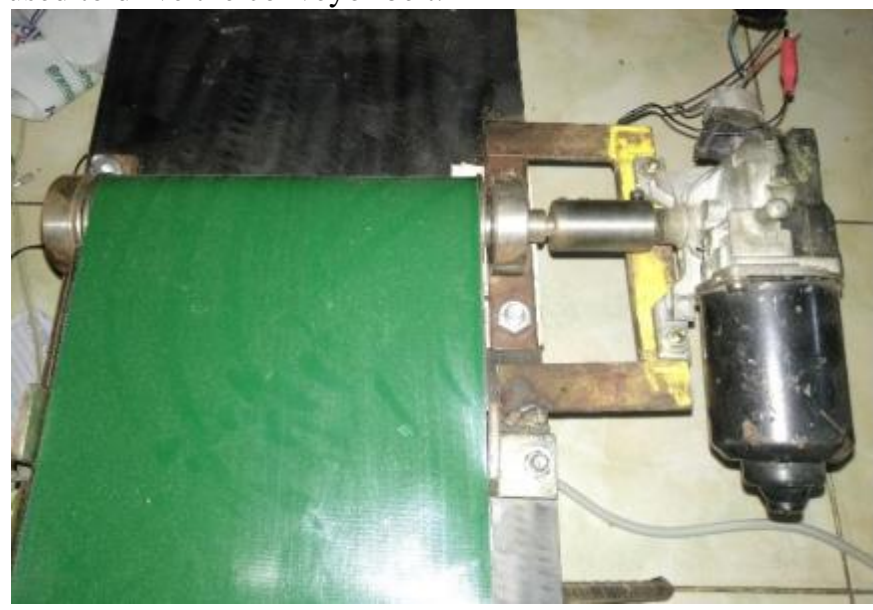

Fig. 9. Implementation of the wiper motor on the system

\section{RESULt ANALYSIS}

The detection and separation results of the system are quite satisfactory. When an object reaches the end of the funnel, two servo motors sweep it to the conveyor. The end of the funnel is made in such a way that it blocks other objects to fall together to the conveyor. Fig. 10(a) shows an object at the 
end of the funnel and fig. 10(b) shows the fall of the object on the conveyor belt by the flaps of the servo motors
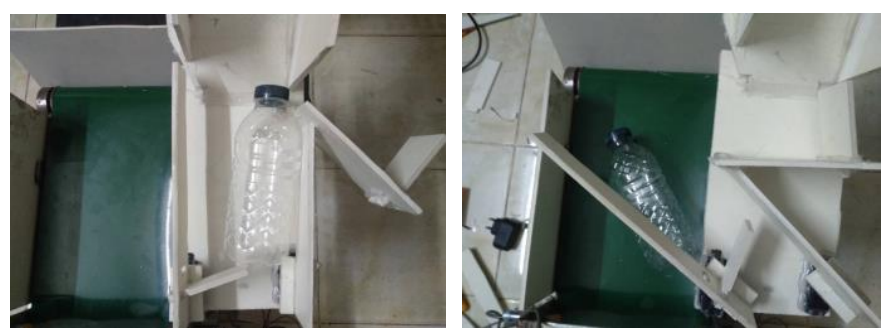

Fig. 10(a) Object at the end of the funnel
Fig. 10(b) Dropping of object on the conveyor from the funnel

Metal is detected by using the inductive proximity sensor. Fig. 11 shows the detection of metal and closing of the gate using a servo motor.

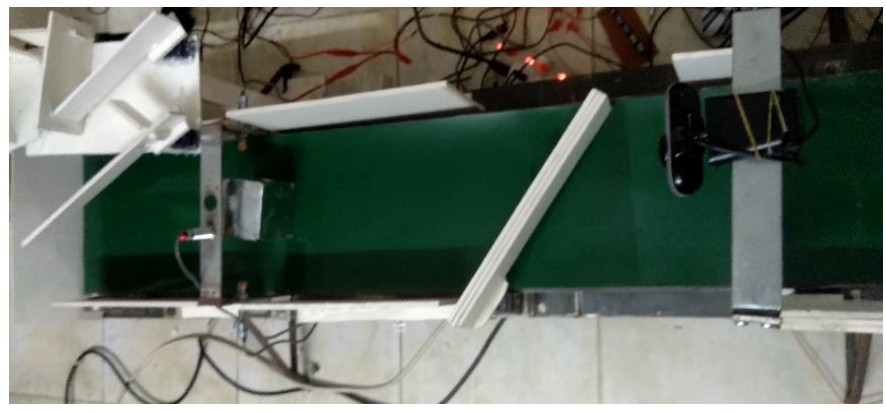

Fig. 11. The detection of metal and closing of the gate using a servo motor

Table II shows the time taken for the detection and separation of metallic waste.

TABLE II. DETECTION AND SEPARATION OF METAL

\begin{tabular}{|c|c|c|}
\hline $\begin{array}{c}\text { Detection and } \\
\text { separation of waste }\end{array}$ & Iteration & Time Taken \\
\hline Metal & 1 & 6 seconds \\
\hline Metal & 2 & 7 seconds \\
\hline Metal & 3 & 6 seconds \\
\hline
\end{tabular}

Other than the metallic objects, the rest of the objects are detected using the machine learning technique. From fig. 12 and fig. 13 show the detection of glass having $98 \%$ confidence accuracy and closing of the flap using machine learning technique respectively.

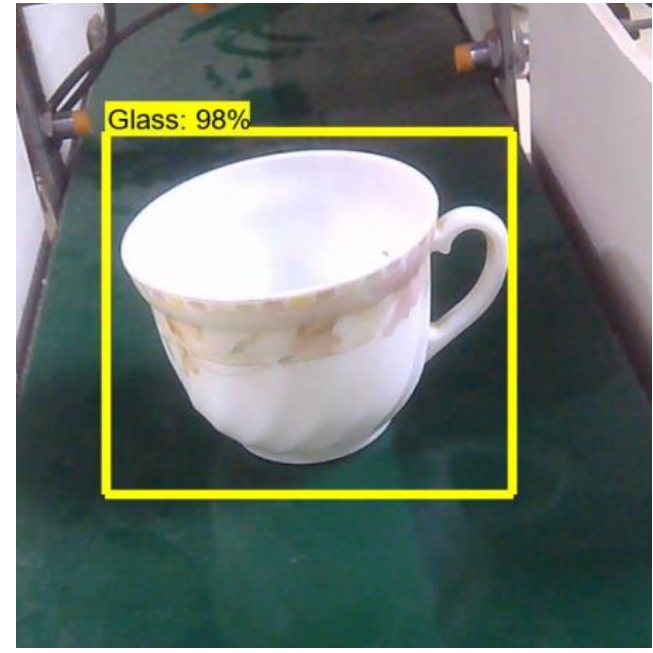

Fig. 12. The detection of glass using machine learning technique

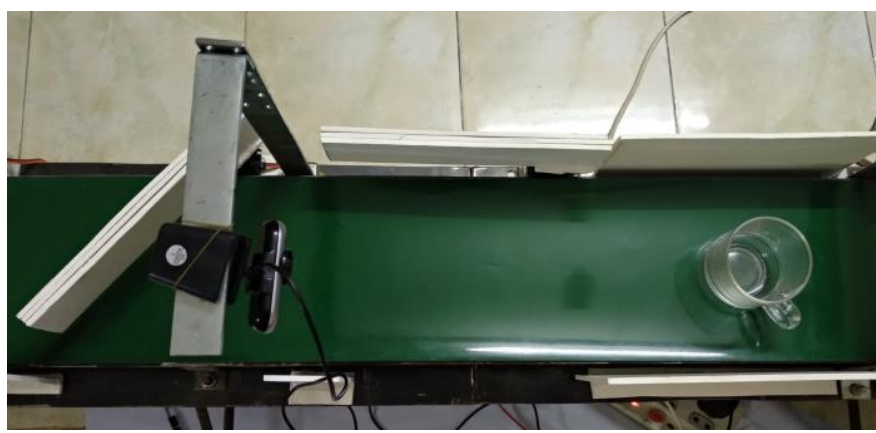

Fig. 13. Closing of the gate after the detection of glass

Table III shows the time taken for the detection and separation of the glass.

TABLE III. DETECTION AND SEPARATION OF GLASS

\begin{tabular}{|c|c|c|}
\hline $\begin{array}{c}\text { Detection and } \\
\text { separation of waste }\end{array}$ & Iteration & Time Taken \\
\hline Glass & 1 & 15 seconds \\
\hline Glass & 2 & 17 seconds \\
\hline Glass & 3 & 16 seconds \\
\hline
\end{tabular}

Fig. 14 shows the detection of the plastic waste having $99 \%$ confidence accuracy.

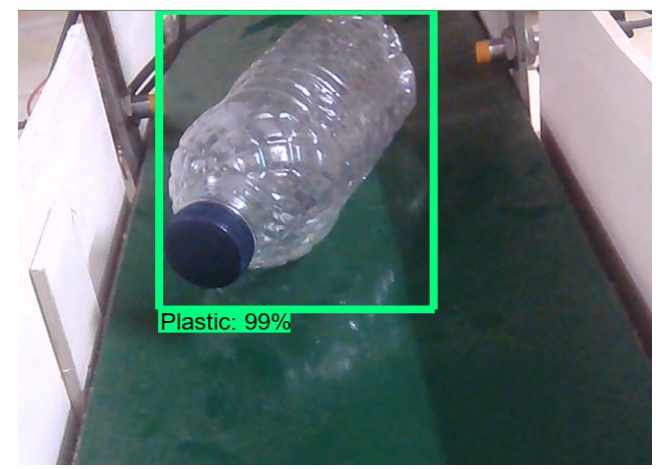

Fig. 14. The detection of plastic using machine learning technique. 
Table IV shows the time taken for the detection and separation of plastic.

TABLE IV. DETECTION AND SEPARATION OF PLASTIC

\begin{tabular}{|c|c|c|}
\hline $\begin{array}{c}\text { Detection and } \\
\text { separation of waste }\end{array}$ & Iteration & Time Taken \\
\hline Plastic & 1 & 21 seconds \\
\hline Plastic & 2 & 19 seconds \\
\hline Plastic & 3 & 20 seconds \\
\hline
\end{tabular}

Fig. 15 shows the detection of the paper waste having $99 \%$ confidence accuracy.

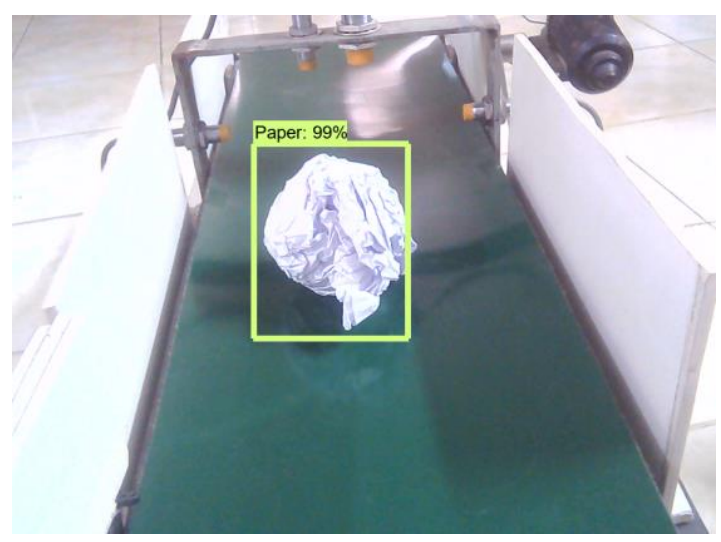

Fig. 15. The detection of paper using machine learning technique

Table $\mathrm{V}$ shows the time taken for the detection and separation of the paper.

TABLE V. DETECTION AND SEPARATION OF PAPER

\begin{tabular}{|c|c|c|}
\hline $\begin{array}{c}\text { Detection and } \\
\text { separation of waste }\end{array}$ & Iteration & Time Taken \\
\hline Paper & 1 & 24 seconds \\
\hline Paper & 2 & 26 seconds \\
\hline Paper & 3 & 25 seconds \\
\hline
\end{tabular}

Fig. 16 shows the detection of the cloth waste having 95\% confidence accuracy.

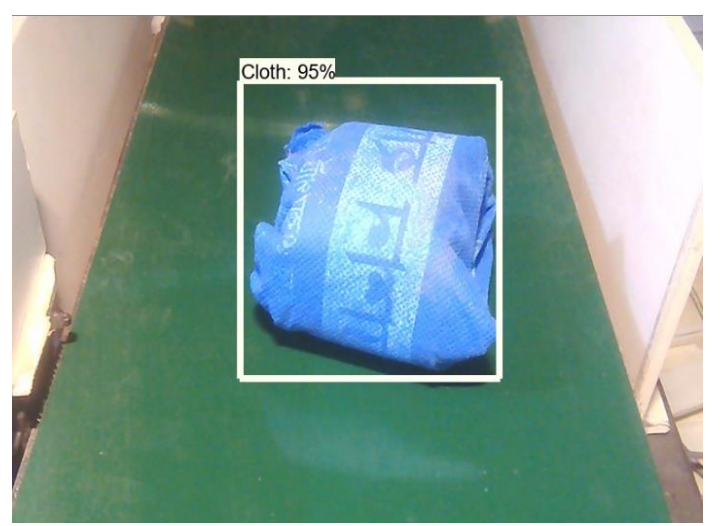

Fig. 16. The detection of cloth using machine learning technique
Table VI shows the time taken for the detection and separation of the cloth.

TABLE VI. DETECTION AND SEPARATION OF CLOTH

\begin{tabular}{|c|c|c|}
\hline $\begin{array}{c}\text { Detection and } \\
\text { separation of waste }\end{array}$ & Iteration & Time Taken \\
\hline Cloth & 1 & 29 seconds \\
\hline Cloth & 2 & 28 seconds \\
\hline Cloth & 3 & 28 seconds \\
\hline
\end{tabular}

Fig. 17 shows the detection of the multiple wastes having high confidence accuracy. They are detected by machine learning techniques using Faster-RCNN method. By using the transfer learning technique an already trained Faster-RCNN method is utilized for fine-tuning and extraction of feature maps to train new data of specific tasks.

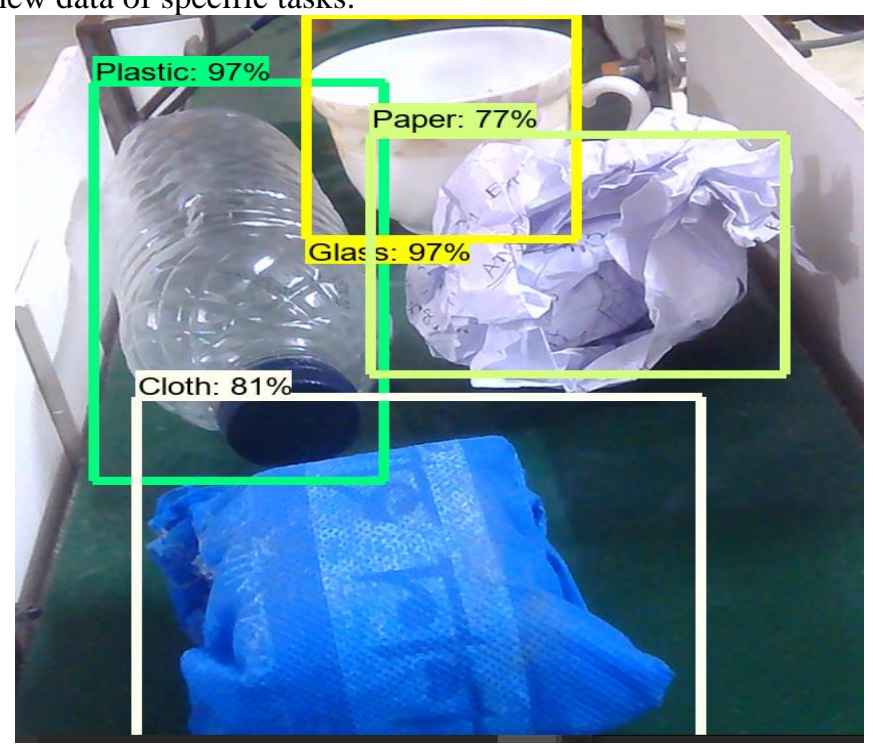

Fig. 17. The detection of multiple objects using trained data

The conveyor belt will stop for three seconds for the camera to detect the object perfectly as the frame per second of the live video feed is in between 1.3 to 1.8 . This could be increased by using a better processing unit. In table VII, the time taken for detecting different types of waste is shown.

TABLE VII. ANALYSIS OF THE RESULT

\begin{tabular}{|c|c|}
\hline $\begin{array}{c}\text { Detection and } \\
\text { separation of waste }\end{array}$ & Time Taken \\
\hline Metal & 6 seconds \\
\hline Glass & 15 seconds \\
\hline Plastic & 19 seconds \\
\hline Paper & 24 seconds \\
\hline Cloth & 28 seconds \\
\hline Unspecified waste & 31 seconds \\
\hline
\end{tabular}

For the detection of metallic objects, it takes the shortest amount of time. For other waste, it needs at least 3 to 4 seconds delay to detect one specific waste due to the limitation 
of the processing speed of the computer. The waste materials, which are not trained to the machine, are not even detected. Hence, they fall at the end of the conveyor and take the longest time to be sorted.

Most of traditional waste sorting systems can separate metallic, dry, and wet waste. But our designed waste sorting machine can even detect and sort out paper, glass, plastic which are very common waste. Plastic and glass can cause clog in the drainage system leading to tremendous problems in the city. These waste materials can be sorted by using the machine learning technique for recycling without any intervention from humans. The building cost of the system is very low. In addition to that, it is possible to train the system with common types of waste material to increase the efficiency of the system. These features are unique for this waste sorting machine.

\section{CONCLUSION}

In today's scenario, recycling is the only sustainable alternative for future generations to come. This project will help to achieve better and more efficient recycling. The main objective of this project is to aid the waste management system in Bangladesh to make a better and safer environment for everyone. Waste products could be reused to save our environment as well as the economic value of the waste materials are so important. Metal supply to our environment is limited. Hence reuse of the metal after successfully sorting out has significant economic values. Moreover, our project focuses on sorting metal, paper, plastic, and glass which are very common types of waste materials in our country. Apart from the short time delay during image processing and the conveyor size- limiting the size of the materials, this work has helped us to create a systematized, labor-saving system. The main advantage of our project is that it is completely automatic and instead of human intervention, computer vision is implemented to sort out waste materials. Waste materials are detrimental to the health of human beings. Hence, instead of manual sorting of waste by humans, our machine can take over this job. Alongside the government, it can be stretched to involve environmental agencies and NGOs to achieve a more energy-efficient and cleaner Bangladesh. Hence, our automatic waste sorting machine has a significant amount of economic, environmental, and social value.

\section{REFERENCES}

[1] A. Ahsan., M. Alamgir, R. Islam, and K. H. Chowdhury. "Initiatives of non-governmental organizations in solid waste management at Khulna City." In Proc. 3rd Annual Paper Meet and Intl. Conf. on Civil Engineering, March, pp. 9-11. 2005.

[2] A. Abbas, R. Nai'ya, and Y.A. Arigbede. "Use of remote sensing and GIS in effective and efficient solid management planning: A case study of Samara, Zaria, Nigeria. Res. J. Earth Planet. Stud”, pp.46-52 2011.

[3] A.A. Mohammedshum, M. Gebresilassiea, C.M Rulindaa, G.H. Kahsaya, and M.S. Tesfay "Application of GIS and Remote Sensing in effective solid waste disposal site selection in Wukro town, Tigray, Ethiopia," The Int. Arch. Photogram. Rem. Sens. Spatial Inform. Sci ,2014.

[4] R. Afroz, K. Hanaki, R. Tudin. "Factors affecting waste generation: A study in a waste management program in Dhaka City, Bangladesh. Environmental Monitoring and Assessment," 2011.
[5] Abedin, M. A., \& Jahiruddin, M. (2015). Waste generation and management in Bangladesh: An overview. Asian Journal of Medical and Biological Research, 1(1), 114-120.

[6] M. Rafeeq, Ateequrahman, S. Alam and Mikdad, "Automation of plastic, metal and glass waste materials segregation using arduino in scrap industry," 2016 International Conference on Communication and Electronics Systems (ICCES), Coimbatore, 2016, pp. 1-5.

[7] Elfasakhany, A., Arrieta, A., Ramírez, D. M., \& Rodríguez, F. (2001). "Design and development of an autonomous trash sorting system." GJ P\&A Sc and Tech. pp, 56-64.

[8] S. M. Samreen, B. Gadgay, V. Pujari, and B.V. Pallvi, "Automatic Metal, Glass and Plastic Waste Sorter", vol. 5, no. 6, pp. 884-889, 2017.

[9] A. Madankar, M. Patil and P. Khandait, "Automated Waste Segregation System and its approach towards generation of Ethanol," 2019 5th International Conference on Advanced Computing \& Communication Systems (ICACCS), Coimbatore, India, 2019, pp. 571-573.

[10] Torres Garcia, Andres \& Rodea-Aragón, Oscar \& Longoria-Gandara, Omar \& Sánchez-García, Francisco \& González-Jiménez, Luis. (2015). "Intelligent Waste Separator." Computacion y Sistemas. 19. 487-500. 10.13053/CyS-19-3-2254.

[11] Sharanya, A., Harika, U., Sriya, N., \& Kochuvila, S. (2017, September). "Automatic waste segregator." In 2017 International Conference on Advances in Computing, Communications and Informatics (ICACCI) (pp. 1313-1319). IEEE.

[12] Sudha, S., Vidhyalakshmi, M., Pavithra, K., Sangeetha, K., \& Swaathi, V. (2016, July). "An automatic classification method for environment: Friendly waste segregation using deep learning." In 2016 IEEE Technological Innovations in ICT for Agriculture and Rural Development (TIAR) (pp. 6570). IEEE.

[13] R. Laszlo, R. Holonec, R. Copîndean and F. Dragan, "Sorting System for e-Waste Recycling using Contour Vision Sensors," 2019 8th International Conference on Modern Power Systems (MPS), Cluj Napoca, Romania, 2019, pp. 1-4.

[14] M. S. Haque Sunny, D. Roy Dipta, S. Hossain, H. M. Resalat Faruque and E. Hossain, "Design of a Convolutional Neural Network Based Smart Waste Disposal System," 2019 1st International Conference on Advances in Science, Engineering and Robotics Technology (ICASERT), Dhaka, Bangladesh, 2019, pp. 1-5.

[15] Szegedy, C., Vanhoucke, V., Ioffe, S., Shlens, J., \& Wojna, Z. (2016). Rethinking the inception architecture for computer vision. In Proceedings of the IEEE conference on computer vision and pattern recognition (pp. 28182826).

[16] C. Jun, Y. Suhua and J. Shaofeng, "Automatic classification and recognition of complex documents based on Faster RCNN," 2019 14th IEEE International Conference on Electronic Measurement \& Instruments (ICEMI), Changsha, China, 2019, pp. 573-577, doi: 10.1109/ICEMI46757.2019.9101847.

[17] "Transfer Learning with TensorFlow Tutorial: Image Classification Example". [Online]. Available: https://lambdalabs.com/blog/transfer-learningwith-tensorflow-tutorial-image-classification-example. [Accessed: 29 April 2020].

[18] "Introduction to Transfer Learning with TensorFlow 2.0". [Online]. Available: https://www.mlq.ai/transfer-learning-tensorflow-2-0. [Accessed: 29 April 2020].

[19] Ren, S., He, K., Girshick, R., \& Sun, J. (2015). Faster r-cnn: Towards real-time object detection with region proposal networks. In Advances in neural information processing systems (pp. 91-99).

[20] "Deep Learning for Object Detection: A Comprehensive Review". [Online]. Available: https://www.kdnuggets.com/2017/10/deep-learningobject-detection-comprehensive-review.html. [Accessed: 29 April 2020].

[21] "Live Object Detection with the Tensorflow Object Detection API". [Online]. Available: https://gilberttanner.com/blog/live-object-detection. [Accessed: 29 April 2020].

[22] "models/detection_model_zoo.md at master · tensorflow/models GitHub". [Online]. Available: https://github.com/tensorflow/models/blob/master/research/object_detection/g 3doc/detection_model_zoo.md. [Accessed: 29 April 2020].

[23] "mAP (mean Average Precision) for Object Detection - Jonathan Hui Medium". [Online]. Available: https://medium.com/@jonathan_hui/map- 
mean-average-precision-for-object-detection-45c121a31173. [Accessed: 29 April 2020].

[24] J. Singh and B. Bhushan, "Real Time Indian License Plate Detection using Deep Neural Networks and Optical Character Recognition using LSTM Tesseract," 2019 International Conference on Computing, Communication, and Intelligent Systems (ICCCIS), Greater Noida, India, 2019, pp. 347-352.

[25] "Train/Test Split and Cross Validation in Python - Towards Data Science". [Online]. Available: https://towardsdatascience.com/train-test-splitand-cross-validation-in-python-80b61beca4b6. [Accessed: 29 April 2020].

[26] Tzutalin, "Labeling tool: LabelImg Git Code," [Online]. Available: https://github.com/tzutalin/labelImg. [Accessed: 29 April 2020].

[27] M. Everingham, L. V. Gool, C. K. I. Williams, J. Winn and A. Zisserman, "The PASCAL Visual Object Classes (VOC) Challenge," International Journal of Computer Vision, vol. 88, no. 2, pp. 303-338, 2010.

[28] "Creating your own object detector - Towards Data Science". [Online]. Available: https://towardsdatascience.com/creating-your-own-object-detectorad69dda69c85. [Accessed: 29 April 2020].

[29] "Freezing a Keras model - Towards Data Science". [Online]. Available: https://towardsdatascience.com/freezing-a-keras-model-c2e26cb84a38.

[Accessed: 29 April 2020].

[30] "TensorBoard | TensorFlow". [Online]. Available: https://www.tensorflow.org/tensorboard. [Accessed: 29 April 2020].

[31] "GitHub - EdjeElectronics/TensorFlow-Object-Detection-API-TutorialTrain-Multiple-Objects-Windows-10: How to train a TensorFlow Object Detection Classifier for multiple object detection on Windows". [Online]. Available: https://github.com/EdjeElectronics/TensorFlow-Object-DetectionAPI-Tutorial-Train-Multiple-Objects-Windows-10. [Accessed: 29 April 2020].

[32] R. Ravindran, M. J. Santora, M. Faied and M. Fanaei, "Traffic Sign Identification Using Deep Learning," 2019 International Conference on Computational Science and Computational Intelligence (CSCI), Las Vegas, NV, USA, 2019, pp. 318-323.

[33] "Common Loss functions in machine learning - Towards Data Science". [Online]. Available: https://towardsdatascience.com/common-loss-functionsin-machine-learning-46afoffc4d23. [Accessed: 29 April 2020].

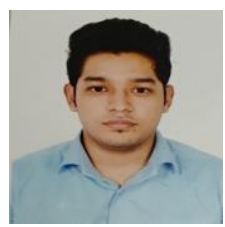

Shirazush Salekin Chowdhury has received his B.Sc. in Electrical and Electronic Engineering (EEE) from American International University-Bangladesh (AIUB) in 2019. He is also pursuing his M.Sc. in EEE from AIUB. Mr. Chowdhury is a graduate student member of the Institute of Electrical and Electronics Engineers (IEEE). Current, he is working a design engineer in Ulkasemi Pvt. Limited. His research interest includes artificial intelligence, VLSI circuit design, semiconductor device, etc.

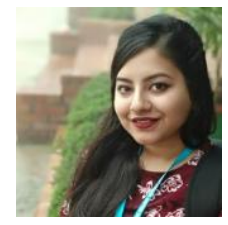

Nahian Binte Hossain has completed her B.Sc. in Electrical and Electronics Engineering in 2020 from American International University-Bangladesh (AIUB). Currently, she is working as a VLSI engineer in Ulkasemi Pvt. Limited. Her research interest includes VLSI circuits and Biomedical electronics.

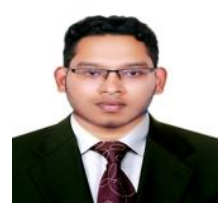

Tamal Saha was born in $8^{\text {th }}$ August 1998. He passed his SSC and HSC from BPATC School and College in 2013 and 2015 respectively. He received his B.Sc. in Electrical and Electronics Engineering from American International University-Bangladesh (AIUB) in January, 2020.

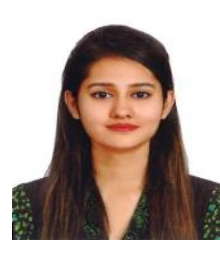

Jannatul Ferdous was a student of American International University-Bangladesh (AIUB) in EEE. She finished her bechelor's degree in 2019 and HSC in 2014. She has participated in a couple of seminars and workshops during her undergraduate program. She was working as a public relations coordinator in AIUB Community of Engineering Students-ACES. Currently, she is working under a freight forwarding company.

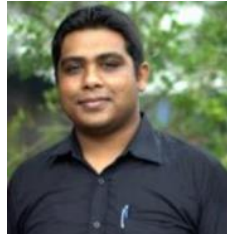

Md. Saniat Rahman Zishan received B.Sc. in Electrical and Electronic Engineering and Master of Engineering in Telecommunications degree from American International University-Bangladesh (AIUB). On September 2009, he started his teaching career as a lecturer in AIUB. At present he is serving as an Associate Professor at the Department of Electrical and Electronic Engineering (EEE) \& Computer Engineering (CoE) of AIUB. He is also serving as the Head of CoE Department. He is enrolled for PhD Degree at Universiti Sultan Zainal Abidin, Malaysia. Mr. Zishan is a member of the Institute of Electrical and Electronics Engineers (IEEE) and Institution of Engineers, Bangladesh (IEB). His current research interest includes Wireless Communication, Signal Processing, E-Health System, Telemedicine, Robotics and AI. 